\title{
Erratum: Interactions of astrophysical neutrinos with dark matter: a model building perspective
}

\author{
Sujata Pandey, Siddhartha Karmakar ${ }^{1}$ and Subhendu Rakshit \\ Discipline of Physics, Indian Institute of Technology Indore, \\ Khandwa Road, Simrol, Indore, 453 552, India \\ E-mail: phd1501151007@iti.ac.in, phd1401251010@iti.ac.in, \\ rakshit@iiti.ac.in
}

ERRATUM TO: JHEP01(2019)095

ArXiv EPRINT: 1810.04203

\section{Introduction}

In ref. [1] we studied whether the neutrino-dark matter (DM) interactions can cause a suppression of astrophysical neutrino flux. We singled out the interactions which reduce the neutrino flux by $\gtrsim 1 \%$, dubbed as 'significant flux suppression' throughout the paper. In light of the collider and electroweak precision constraints, we concluded that out of the eleven effective and three renormalisable neutrino-DM interactions studied in ref. [1], three could still lead to at least $1 \%$ suppression of astrophysical neutrino flux at IceCube. These three scenarios involve ultralight scalar DM interacting with neutrinos through $(i)$ Topology I 3 from eq. (3.3), (ii) Topology III in section 3.3, and (iii) vector-mediated interaction in section 4.3 .3 of ref. [1].

In this erratum we add that, as the Big Bang Nucleosynthesis (BBN) constraints forbid neutrinos to be in thermal equilibrium with light scalar DM after the neutrino decoupling epoch, two out of the aforementioned three scenarios fail to lead to any significant flux suppression. To be precise, Topology III in section 3.3 and vector-mediated interaction in section 4.3.3 cannot lead to significant neutrino flux suppression, while Topology I 3 still can. The BBN bounds on these three scenarios are discussed in a more detail in the next section.

\footnotetext{
${ }^{1}$ Now at: Department of Physics, Indian Institute of Technology Bombay, Powai, Mumbai, 400 076, India; karmakars@iitb.ac.in.
} 


\section{Details of BBN bounds on neutrino-DM interactions}

To satisfy the Big Bang Nucleosynthesis (BBN) constraints on neutrino interactions with ultralight scalar DM $\Phi$, the rate of $\nu \nu \leftrightarrow \Phi \Phi$ at the neutrino decoupling temperature $T_{\nu} \sim 2 \mathrm{MeV}$ must be less than Hubble expansion rate $H$. Thus, at $T_{\nu}$, the cross-section of $\nu \nu \leftrightarrow \Phi \Phi$ has to be $\lesssim H / n_{\nu} \sim 3.5 \times 10^{-34} \mathrm{eV}^{-2}$. For the interaction given by Topology I3,

$$
\mathcal{L} \supset \frac{c_{l}^{(3)}}{\Lambda} \bar{\nu}^{c} \nu \Phi^{\star} \Phi
$$

the aforementioned bound translates to $c_{l}^{(3)} / \Lambda \lesssim 2.5 \times 10^{-5} \mathrm{GeV}^{-1}$ in the limit $m_{\Phi} \ll T_{\nu}$. For the maximally allowed value of the coupling strength, $c_{l}^{(3)} / \Lambda=2.5 \times 10^{-5} \mathrm{GeV}^{-1}$, this interaction can lead to $1 \%$ flux suppression of neutrinos at IceCube, i.e., $\exp \left(-n_{\mathrm{DM}} \sigma_{\nu \mathrm{DM}} L\right) \sim$ 0.01 , if $m_{\mathrm{DM}} \lesssim 4 \times 10^{-8} \mathrm{eV}$. Here, we consider the length traversed by neutrinos $L=200 \mathrm{Mpc}$ and isotropic DM density $\rho_{\mathrm{DM}}=n_{\mathrm{DM}} m_{\mathrm{DM}} \sim 1.2 \times 10^{-6} \mathrm{GeV} \mathrm{cm}{ }^{-3}$, as mentioned on pg. 4 of ref. [1]. For Topology I 3, the neutrino-DM scattering cross-section $\sigma_{\nu \mathrm{DM}}$ is independent of DM mass, but neutrino flux suppression still depends on the value of $m_{\mathrm{DM}}$ as lighter DM would imply a larger value of DM number density.

Contrary to Topology I 3 , there are models for which $\nu$-DM scattering cross-section increases with $m_{\mathrm{DM}}$, as shown in figures $3(\mathrm{~b})$ and $8(\mathrm{~b})$ of ref. [1]. BBN constraint on such models disfavours neutrino flux suppression for the entire range of ultralight scalar DM mass. For instance, in Topology III, i.e.,

$$
\mathcal{L} \supset C_{1}\left(\Phi^{*} \partial_{\mu} \Phi-\Phi \partial_{\mu} \Phi^{*}\right) Z^{\prime \mu}+\frac{c_{l}^{(9)}}{\Lambda}\left(\bar{\nu}^{c} \sigma_{\mu \nu} P_{L} \nu\right) Z^{\prime \mu \nu},
$$

the BBN bound reads $C_{1} c_{l}^{(9)} / \Lambda \lesssim 2.5 \times 10^{-6} \mathrm{GeV}^{-1}$, which disfavours any significant flux suppression.

The BBN constraint on renormalisable vector mediated $\nu$-DM interaction,

$$
\mathcal{L} \supset f_{l}^{\prime} \bar{l} \gamma^{\mu} P_{L} l Z_{\mu}^{\prime}+i g^{\prime}\left(\Phi^{*} \partial^{\mu} \Phi-\Phi \partial^{\mu} \Phi^{*}\right) Z_{\mu}^{\prime},
$$

reads $f_{l}^{\prime} g^{\prime} \lesssim 6 \times 10^{-8}$ for $m_{Z^{\prime}} \sim 10 \mathrm{MeV}$. This does not allow for any significant change in the astrophysical neutrino flux. Though this interaction can still lead to changes in the flavour of astrophysical neutrinos passing through solitonic core of ultralight DM [2].

The key constraints on the effective and renormalisable interactions for light DM are summarised in tables 1 and 2 below. For DM with higher masses, the cosmological constraints such as, relic density, collisional damping, and $N_{\text {eff }}$ ensure that the mentioned interactions do not lead to any significant flux suppression, as shown in figure 5 of ref. [1].

\section{Conclusion}

In this erratum we point out, Topology III and the renormalisable vector-mediated model of neutrino-DM interactions are already too constrainted by BBN to show up at the IceCube neutrino observatory. Only Topology I 3 can lead to a suppression of astrophysical neutrino flux even after imposing the BBN bounds. Tables 1 and 2 are similar to tables 4 and 5 of ref. [1] respectively, but are improved with these BBN bounds. 


\begin{tabular}{|c|c|c|c|}
\hline Topology & Interaction & Constraints & Remarks \\
\hline I 1 & $\frac{c_{l}^{(1)}}{\Lambda^{2}}(\bar{\nu} i \not \partial \nu)\left(\Phi^{*} \Phi\right)$ & $\begin{array}{l}c_{l}^{(1)} / \Lambda^{2} \lesssim 8.8 \times 10^{-3} \mathrm{GeV}^{-2}, c_{e}^{(1)} / \Lambda^{2} \lesssim 1.0 \times 10^{-4} \mathrm{GeV}^{-2}, \\
c_{\mu}^{(1)} / \Lambda^{2} \lesssim 6.0 \times 10^{-3} \mathrm{GeV}^{-2}, c_{\tau}^{(1)} / \Lambda^{2} \lesssim 6.2 \times 10^{-3} \mathrm{GeV}^{-2}\end{array}$ & disfavoured \\
\hline I 2 & $\begin{array}{l}\frac{c_{l}^{(2)}}{\Lambda^{2}}\left(\bar{\nu} \gamma^{\mu} \nu\right)\left(\Phi^{*} \partial_{\mu} \Phi\right. \\
\left.\quad-\Phi \partial_{\mu} \Phi^{*}\right)\end{array}$ & $\begin{array}{l}c_{l}^{(2)} / \Lambda^{2} \lesssim 1.8 \times 10^{-2} \mathrm{GeV}^{-2}, c_{e}^{(2)} / \Lambda^{2} \lesssim 2.6 \times 10^{-5} \mathrm{GeV}^{-2}, \\
c_{\mu}^{(1)} / \Lambda^{2} \lesssim 1.2 \times 10^{-2} \mathrm{GeV}^{-2}, c_{\tau}^{(1)} / \Lambda^{2} \lesssim 1.3 \times 10^{-3} \mathrm{GeV}^{-2}\end{array}$ & disfavoured \\
\hline I 3 & $\frac{c_{l}^{(3)}}{\Lambda} \bar{\nu}^{c} \nu \Phi^{\star} \Phi$ & $c_{l}^{(3)} / \Lambda \leq 0.5 \mathrm{GeV}^{-1}, c_{l}^{(3)} / \Lambda \lesssim 2.5 \times 10^{-5} \mathrm{GeV}^{-1}$ & favoured $^{a}$ \\
\hline I 4 & $\begin{array}{l}\frac{c_{l}^{(4)}}{\Lambda^{3}}\left(\overline{\nu^{c}} \sigma^{\mu \nu} \nu\right)\left(\partial_{\mu} \Phi^{*} \partial_{\nu} \Phi\right. \\
\left.-\partial_{\nu} \Phi^{*} \partial_{\mu} \Phi\right)\end{array}$ & $c_{l}^{(4)} / \Lambda^{3} \lesssim 2.0 \times 10^{-3} \mathrm{GeV}^{-3}$ & disfavoured \\
\hline I 5 & $\frac{c_{l}^{(5)}}{\Lambda^{3}} \partial^{\mu}\left(\bar{\nu}^{c} \nu\right) \partial_{\mu}\left(\Phi^{*} \Phi\right)$ & $c_{l}^{(5)} / \Lambda^{3} \lesssim 7.5 \times 10^{-4} \mathrm{GeV}^{-3}$ & disfavoured \\
\hline I 6 & $\begin{array}{l}\frac{c_{l}^{(6)}}{\Lambda^{4}}\left(\bar{\nu} \partial^{\mu} \gamma^{\nu} \nu\right)\left(\partial_{\mu} \Phi^{*} \partial_{\nu} \Phi\right. \\
\left.\quad-\partial_{\nu} \Phi^{*} \partial_{\mu} \Phi\right)\end{array}$ & $\begin{array}{c}c_{l}^{(6)} / \Lambda^{4} \lesssim 2.5 \times 10^{-5} \mathrm{GeV}^{-4}, c_{e}^{(6)} / \Lambda^{4} \lesssim 1.2 \times 10^{-6} \mathrm{GeV}^{-4} \\
c_{\mu}^{(6)} / \Lambda^{4} \sim c_{\tau}^{(6)} / \Lambda^{4} \lesssim 10^{-5} \mathrm{GeV}^{-4}\end{array}$ & disfavoured \\
\hline II 1 & $\begin{aligned} & \frac{c_{l}^{(7)}}{\Lambda^{2}}\left(\partial^{\mu} \Phi^{*} \partial^{\nu} \Phi\right. \\
- & \left.\partial^{\nu} \Phi^{*} \partial^{\mu} \Phi\right) Z_{\mu \nu}^{\prime} \\
+ & f_{i} \bar{\nu}_{i} \gamma^{\mu} P_{L} \nu_{i} Z_{\mu}^{\prime}\end{aligned}$ & $\begin{array}{c}f_{l} c_{l}^{(7)} / \Lambda^{2} \lesssim 4.2 \times 10^{-2} \mathrm{GeV}^{-2}, f_{e} c_{e}^{(7)} / \Lambda^{2} \lesssim 1.9 \times 10^{-5} \mathrm{GeV}^{-2} \\
f_{\mu} c_{\mu}^{(7)} / \Lambda^{2} \sim f_{\tau} c_{\tau}^{(7)} / \Lambda^{2} \lesssim 8.1 \times 10^{-3} \mathrm{GeV}^{-2} \\
{\left[f_{e}, f_{\mu}, f_{\tau}\right] \lesssim\left[10^{-5}, 10^{-6}, 0.02\right] \text { for } m_{Z^{\prime}} \sim 10 \mathrm{MeV}}\end{array}$ & disfavoured \\
\hline II 2 & $\frac{c_{l}^{(8)}}{\Lambda} \partial^{\mu}|\Phi|^{2} \partial_{\mu} \Delta+f_{l} \overline{\nu^{c}} \nu \Delta$ & $m_{\nu} \sim f_{l} v_{\Delta} \lesssim 0.1 \mathrm{eV}, m_{\Delta} \gtrsim 150 \mathrm{GeV}$ & disfavoured \\
\hline III & $\begin{array}{l}C_{1}\left(\Phi^{*} \partial_{\mu} \Phi-\Phi \partial_{\mu} \Phi^{*}\right) Z^{\prime \mu} \\
+\frac{c_{l}^{(9)}}{\Lambda}\left(\overline{\nu^{c}} \sigma_{\mu \nu} P_{L} \nu\right) Z^{\prime \mu \nu}\end{array}$ & $\begin{array}{c}C_{1} c_{l}^{(9)} / \Lambda \lesssim 3.8 \times 10^{-3} \mathrm{GeV}^{-1} \text { and } C_{1} c_{l}^{(9)} / \Lambda \lesssim 2.5 \times 10^{-6} \mathrm{GeV}^{-1} \\
\text { for } m_{Z^{\prime}} \sim 10 \mathrm{MeV}\end{array}$ & disfavoured \\
\hline IV & $\frac{c_{l}^{(10)}}{\Lambda^{2}} \bar{L} F_{R} \Phi|H|^{2}+C_{L} \bar{L} F_{R} \Phi$ & Same as in fermion case in table 5 & disfavoured \\
\hline
\end{tabular}

${ }^{a}$ Disfavoured if realised with a $\mathrm{SU}(2)_{L}$ triplet scalar. Also disfavoured for $m_{\mathrm{DM}} \gtrsim 4 \times 10^{-8} \mathrm{eV}$.

Table 1. Summary of neutrino-DM effective interactions. $c_{l}$ and $c_{e, \mu, \tau}$ represent the coefficients of interactions for the gauge non-invariant and gauge-invariant forms respectively. The colour coding for the constraints is: $Z \rightarrow i n v$, LEP monophoton $+\mathbb{E}_{T}, Z \rightarrow \mu^{+} \mu^{-}, Z \rightarrow \tau^{+} \tau^{-}$, BBN and $(g-2)_{e, \mu}$. We also remark whether the interactions are favoured in context of the $1 \%$ flux suppression criteria.

\begin{tabular}{|c|c|c|c|}
\hline Mediator & Interaction & Constraints & Remarks \\
\hline Fermion & $\left(C_{L} \bar{L} F_{R}+C_{R} \bar{l}_{R} F_{L}\right) \Phi+$ & $m_{F} \gtrsim 100 \mathrm{GeV}, m_{\mathrm{DM}} \gtrsim 10^{-21} \mathrm{eV}$, & disfavoured \\
& $h . c$. & $C_{L} C_{R} \lesssim\{2.5,0.5\} \times 10^{-5}$ for $e$ and $\mu$ & \\
\hline Scalar & $f_{l} \bar{L}^{c} L \Delta+g_{\Delta} \Phi^{*} \Phi|\Delta|^{2}$ & $m_{\nu} \sim f_{l} v_{\Delta} \lesssim 0.1 \mathrm{eV}, g_{\Delta} \sim v_{\Delta}^{2} / m_{\mathrm{DM}}^{2}$ & disfavoured \\
\hline Vector & $f_{l}^{\prime} \bar{L} \gamma^{\mu} P_{L} L Z_{\mu}^{\prime}+i g^{\prime}\left(\Phi^{*} \partial^{\mu} \Phi\right.$ & {$\left[f_{e}^{\prime}, f_{\mu}^{\prime}, f_{\tau}^{\prime}\right] \lesssim\left[10^{-5}, 10^{-6}, 0.02\right]$ and $f^{\prime} g^{\prime} \lesssim 6 \times 10^{-8}$ for } & disfavoured \\
& $\left.-\Phi \partial^{\mu} \Phi^{*}\right) Z_{\mu}^{\prime}$ & $m_{Z^{\prime}} \sim 10 \mathrm{MeV}$ & \\
\hline
\end{tabular}

Table 2. Summary of renormalisable neutrino-DM interactions. Colour coding is the same as in table 1 .

Overall, our key conclusion remains the same: building effective and renormalisable models of neutrino-DM interactions which can lead to significant neutrino flux suppression at IceCube is rather hard when confronted with the existing precision, collider, astrophysical, and cosmological constraints. 
Open Access. This article is distributed under the terms of the Creative Commons Attribution License (CC-BY 4.0), which permits any use, distribution and reproduction in any medium, provided the original author(s) and source are credited.

\section{References}

[1] S. Pandey, S. Karmakar and S. Rakshit, Interactions of Astrophysical Neutrinos with Dark Matter: A model building perspective, JHEP 01 (2019) 095 [arXiv: 1810.04203] [INSPIRE].

[2] S. Karmakar, S. Pandey and S. Rakshit, Astronomy with energy dependent flavour ratios of extragalactic neutrinos, JHEP 10 (2021) 004 [arXiv:2010.07336] [INSPIRE]. 